\title{
A randomized trial of symptom-based management in Japanese patients with COPD
}

This article was published in the following Dove Press journal: International Journal of COPD

\author{
Tomoko Betsuyaku' \\ Motokazu Kato ${ }^{2}$ \\ Keisaku Fujimoto ${ }^{3}$ \\ Akihiro Kobayashi ${ }^{4}$ \\ Tomoyuki Hayamizu ${ }^{4}$ \\ Hideki Hitosugi ${ }^{4}$ \\ Gerald Hagan ${ }^{5}$ \\ Mark $\mathrm{H}$ James ${ }^{6}$ \\ Paul W Jones ${ }^{6}$ \\ 'Division of Pulmonary Medicine, \\ Department of Medicine, Keio \\ University, Tokyo, Japan; ${ }^{2}$ Chest \\ Disease Clinical and Research \\ Institute, Kishiwada City Hospital, \\ Kishiwada, Japan; ${ }^{3}$ Department of \\ Clinical Laboratory Sciences, Shinshu \\ University, Matsumoto, Japan; ${ }^{4} \mathrm{GSK}$ \\ K.K., MA, Respiratory, Tokyo, Japan; \\ ${ }^{5}$ Respiratory Science Consultant, \\ Marbella, Spain; ${ }^{6}$ GSK, Respiratory \\ Franchise Medical, GSK House, \\ Brentford, Middlesex, UK
}

\begin{abstract}
Background: The Global initiative for chronic Obstructive Lung Disease strategy document for COPD recommends treatment changes according to the persistence of symptoms or exacerbations. This study assessed the feasibility and outcomes of a structured step-up/step-down treatment approach in a randomized controlled clinical trial setting.

Methods: Japanese patients with moderate-to-severe COPD were randomized to blinded, double-dummy treatment with twice-daily fluticasone propionate/salmeterol (FP/SAL) $250 / 50 \mu \mathrm{g}$ or once-daily tiotropium bromide (TIO) $18 \mu \mathrm{g}$ for 24 weeks (dual bronchodilator was not available). At 4-weekly intervals, patients remaining symptomatic (COPD Assessment Test score $>10$ ) or experiencing an exacerbation were offered the option to use triple therapy. Primary endpoint was the proportion of patients remaining on randomized therapy.

Results: In total, 406 patients participated (mean $\mathrm{FEV}_{1}$ 59\% $\pm 13 \%$ predicted; COPD Assessment Test 12 \pm 6 ). Of these, 204 and 201 patients were included in the FP/SAL and TIO groups, respectively, of whom $67 \%$ and $63 \%$ continued treatment throughout the study; this difference was not statistically significant. Time to first therapy switch was longer with FP/SAL, but not significantly $(P=0.21)$. More patients in Global initiative for chronic Obstructive Lung Disease (2011 criteria) groups C/D switched (FP/SAL 55\%, TIO 63\%) than in groups A/B (FP/SAL 27\%, TIO 27\%).

Conclusion: Given the choice, patients with more symptoms or those experiencing an exacerbation will agree to step-up therapy. Effectiveness of disease management pathways can be tested using double-blind studies.
\end{abstract}

Keywords: COPD management, fluticasone propionate/salmeterol, tiotropium

\section{Introduction}

COPD is a disease characterized by chronic airflow limitation and airways inflammation, and remains a major health care problem. ${ }^{1}$ While there is no cure for COPD, it is preventable and treatable by reducing the impact of symptoms, such as cough, sputum production, and dyspnea, and reducing the risk of exacerbations. ${ }^{1}$

Guidelines, including the Global initiative for chronic Obstructive Lung Disease (GOLD) strategy and national guidelines, suggest a stepped approach to pharmacologic management of COPD following treatment algorithms or pathways in which treatment is changed according to the patient's needs. ${ }^{1-3}$ For example, the 2017 GOLD strategy describes treatment pathways that entail treatment intensification by combining different classes of agents; however, these recommendations are not accompanied by guidance as to when these new treatments should be added. ${ }^{1}$ The guidelines suggest that treatment should be stepped up if the patient has "persisting symptoms" or "further exacerbations", but do not define what constitutes either of these clinical states. Similarly, other guidelines, such as those of the Japanese Respiratory Society, suggest a progressive addition of treatment, again without specific guidance as to when to step up treatment. ${ }^{2}$
Correspondence: Paul W Jones

GSK, Respiratory Franchise Medical,

GSK House, Brentford, Middlesex

TW8 9GS, UK

Email paul.8.jones@gsk.com (c) (i) (c) 2018 Betsuyaku et al. This work is published and licensed by Dove Medical Press Limited. The full terms of this license are available at https://www.dovepress.com/terms.php cc. hereby accept the Terms. Non-commercial uses of the work are permitted without any further permission from Dove Medical Press Limited, provided the work is properly attributed. For permission for commercial use of this work, please see paragraphs 4.2 and 5 of our Terms (https://www.dovepress.com/terms.php). 
Very few studies have tested stepping up (or stepping down) treatment, although one exception is the WISDOM study that was designed to test withdrawal of inhaled corticosteroids (ICS). ${ }^{4}$ Another issue is that most trials are parallel-group comparisons in which the treatment is determined by the randomization process rather than by the treating physician.

The majority of clinical studies used to inform guidelines are efficacy trials designed to test the benefit of one treatment compared with another under standardized conditions. Also, guidelines need to be informed by trials that test treatment pathways that allow the patient and physician to exercise treatment choices, and reflect routine practice more closely.

This study, COPD Symptom-based Management to Optimize Treatment Strategy in Japan (COSMOS-J), was conducted to evaluate the feasibility of a randomized controlled trial (RCT) designed to test a symptom-based pharmacological treatment pathway using a step-up (step-down) to (from) triple therapy. The study was conducted in Japanese patients with moderate-to-severe COPD using a unique protocol, which was based on monthly assessment of symptoms using the COPD Assessment Test (CAT) and monitoring the occurrence of exacerbations since the previous study visit. The treatment approach was consistent with the Japanese licenses for the drugs used and the Japanese Respiratory Society guidelines at the time of protocol development. ${ }^{2}$ It was also consistent with Japanese clinical practice that requires the patient to request maintenance treatment from their physician, since a repeat-prescription service is not available. When the study was designed (October 2012), dual bronchodilators were not available in Japan; therefore, a long-acting muscarinic antagonist (LAMA) and the combination ICS/long-acting $\beta_{2}$-agonist (LABA) were used as randomized therapy, with the possibility to step up to triple therapy (ICS/LABA/LAMA). The rationale for these choices was that LAMA and ICS/LABA both improve St George's Respiratory Questionnaire (SGRQ) scores, ${ }^{5,6}$ and triple therapy has been associated with improvements in outcomes such as exacerbations and health status, whether LAMA is added to ICS/LABA or vice versa. ${ }^{7-13}$ Triple therapy has also been shown to be associated with lower all-cause mortality and oral corticosteroid bursts compared with ICS/LABA. ${ }^{14}$

Some of the results of this study have been previously reported in abstract form. ${ }^{15}$

\section{Methods}

This was a 24-week, multicenter, randomized, blinded, doubledummy study performed in Japan (GSK study number: SCO116717; ClinicalTrials.gov identifier: NCT01762800). The study was conducted in accordance with International Conference on Harmonisation-Good Clinical Practice and the Declaration of Helsinki (2008). All patients provided written informed consent prior to participation in the study. The ethics and review boards of all participating institutions approved the protocol prior to commencement of the study (Table S1). Anonymized individual participant data and study documents can be requested for further research from www.clinicalstudydatarequest.com.

\section{Patients}

Patients aged 40-80 years with an established clinical history of COPD defined by the GOLD 2011 criteria, ${ }^{16}$ a current or former smoking history of $>10$ pack-years, a post-bronchodilator $\mathrm{FEV}_{1}$ of $\geq 30 \%$ but $\leq 80 \%$ of predicted normal values, a post-bronchodilator $\mathrm{FEV}_{1} / \mathrm{FVC}$ ratio of $<70 \%$ and a grade of $\geq 1$ on the modified Medical Research Council (mMRC) scale were included. Exclusion criteria for entry into the study included a medical diagnosis of predominant asthma or a respiratory disorder other than COPD that might interfere with the study, lung transplantation and/or lung volume reduction, and a requirement for regular or long-term oxygen therapy ( $\geq 12$ hours a day). Patients were classified into one of the four GOLD groups (A-D), using the GOLD 2011 criteria, based on history of exacerbations, airflow limitation, and CAT score.

\section{Study protocol}

The full protocol has been published. ${ }^{17}$ After a 4-week run-in period during which patients remained on their usual treatment for COPD, patients were randomized to receive fluticasone propionate/salmeterol combination (FP/SAL; Adoair $^{\text {TM }}$ GlaxoSmithKline, Brentford, UK) 250/50 $\mu \mathrm{g}$ twice daily delivered via the DISKUS ${ }^{\text {TM }}$ (GlaxoSmithKline) inhalation device ("FP/SAL-single"), or tiotropium bromide (TIO; SPIRIVA ${ }^{\mathrm{TM}}$ [Boehringer Ingelheim, Ingelheim, German]) $18 \mu \mathrm{g}$ delivered once daily via the Handihaler ${ }^{\mathrm{TM}}$ inhalation device ("TIO-single") for 24 weeks. These are the licensed doses for these medicines in Japan. Randomized treatments were given blinded and double dummy, so patients in both groups also received placebo medication via a matched-placebo inhalation device (Figure 1). Those patients who stepped up were therefore unblinded, in that they knew that they were receiving both treatments, but they remained blind to their randomized therapy. Patients who were subsequently treated with triple therapy could receive additional non-trial medication, at their physician's discretion.

After randomization, patients were reviewed every 4 weeks to assess their symptom level and the occurrence of any exacerbations (Figure 1). If they remained symptomatic, as measured using the $\mathrm{CAT},{ }^{18}$ or experienced an exacerbation, 


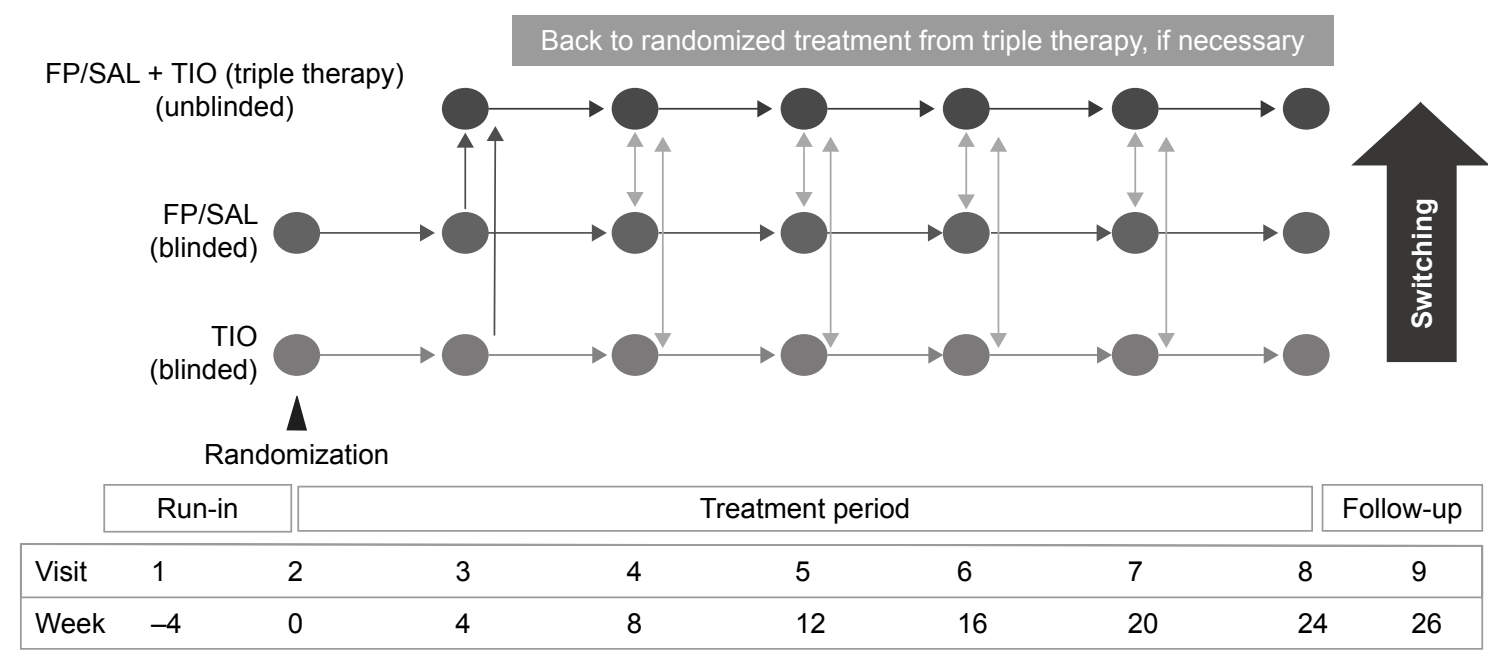

Figure I Study design.

Abbreviations: FP/SAL, fluticasone propionate/salmeterol; TIO, tiotropium.

they were offered the option to step up from randomized treatment to triple therapy. The strength of the recommendation to step up was based on predefined CAT scores as follows: CAT score $<10$, "Your score suggests you are doing well. Are you satisfied with your current treatment?"; CAT score $\geq 10$ but $\leq 15$, "Your score suggests your chest trouble is causing you some problems because of [highest scoring CAT item(s)]. Are you satisfied with your current treatment?"; and CAT score $>15$, "Your score clearly suggests your chest problem is having a big effect on you. We have an option to increase treatment. Do you want to try it?".

Patients were also allowed to step down from triple to randomized treatment. ${ }^{17}$ The procedure for stepping down was based on the judgment of the treating physician and not specified in the protocol. This was largely due to the fact that the study would have been underpowered, for the number of patients who might be stepped down after previous step-up was unknown. In addition, the study duration would have allowed only a limited and very variable time for follow-up of patients following treatment step-down.

\section{Assessments}

The primary endpoint was the proportion of patients who remained on randomized therapy throughout the study. Reported exacerbations and CAT scores were captured at each visit to inform physician and patient choices. Unreported COPD exacerbations were identified using the Exacerbations of COPD Tool (EXACT) diary. ${ }^{19}$ COPD symptoms were measured using EXACT-Respiratory Symptoms (E-RS) scores collected from the EXACT diary. ${ }^{20}$ The physicians did not use the EXACT diaries in their assessment. The proportion of patients who remained on randomized therapy and the proportion of patients who stepped down according to predefined criteria were recorded. For the subgroup analysis, patients were categorized using the GOLD 2011 assessment scheme, with the CAT score as the cut point for low and high symptoms.

Safety was measured by recording any adverse events (AEs) and COPD exacerbations.

\section{Statistical analysis}

It was assumed that the switch rate would be between $10 \%$ and $60 \%$. Using a planned sample size of 400 patients, a $15 \%$ difference would be detectable with $86 \%-99 \%$ power by a log-rank test. The proportion of patients remaining on randomized therapy was estimated using a binomial distribution, and the time to switch to triple therapy was tested using Kaplan-Meier plots with log-rank tests.

To investigate factors that influenced the switch to triple therapy, univariate and multiple logistic regression analyses were conducted using the following covariates: randomized therapy, GOLD 2011 group, sex, age, baseline RS-subscale scores, and eosinophil category.

\section{Results}

Of the 570 patients screened, 406 were randomized (Figure 2). One patient in the TIO group did not receive randomized medication, and so the analysis was performed on the modified intent-to-treat population (TIO: $\mathrm{n}=201$; FP/SAL: $\mathrm{n}=204$; Figure 2). The reasons for patient discontinuation are shown in Figure 2. Very few patients withdrew during the study period with $366(90.1 \%)$ patients remaining in the study. The main reason for withdrawal was experiencing an AE. Patient baseline characteristics are shown in Table 1; 


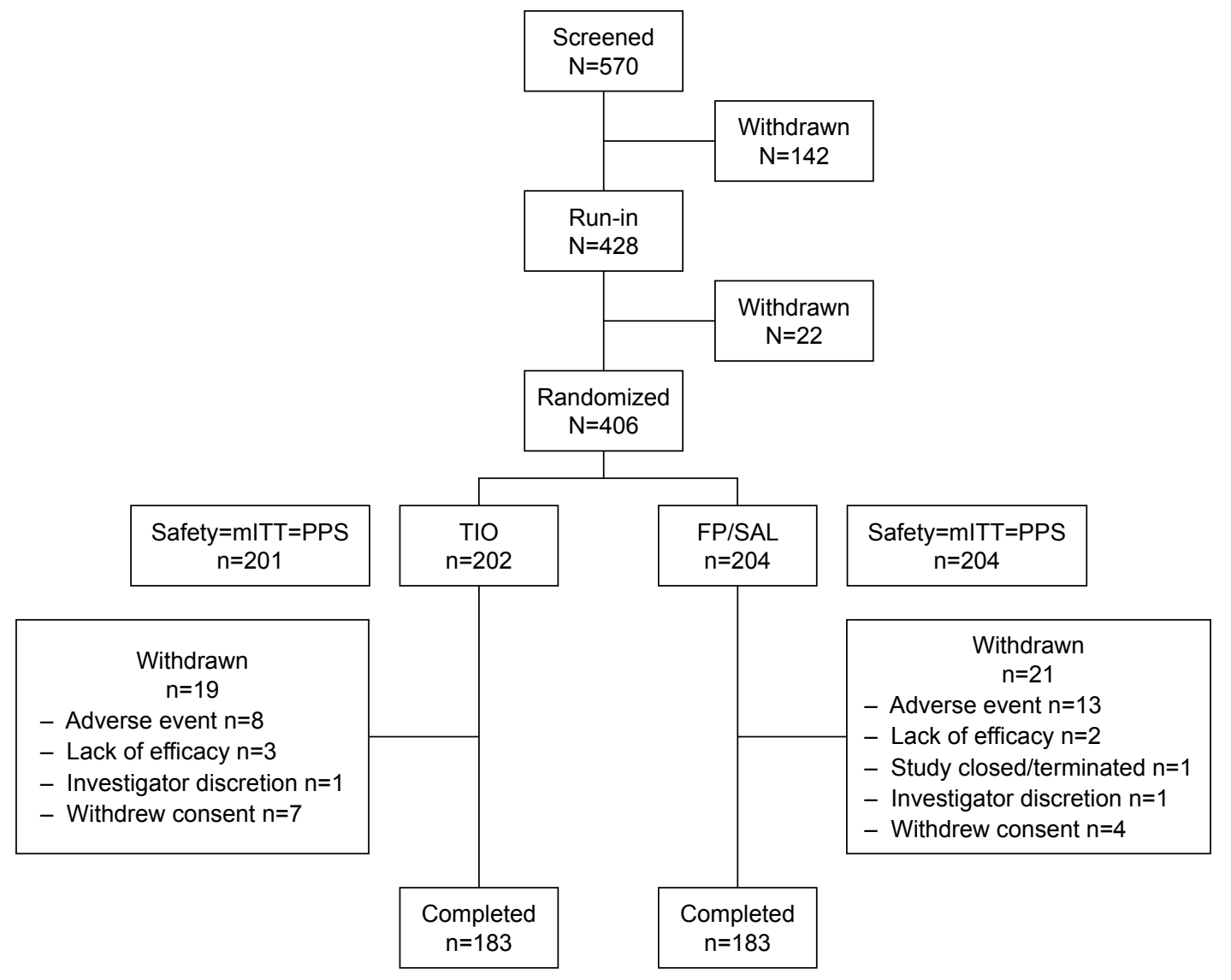

Figure 2 Consort diagram.

Note: mITT: one patient was removed from the TIO group for not receiving study medication.

Abbreviations: FP/SAL, fluticasone propionate/salmeterol; mITT, modified intent-to-treat; PPS, per-protocol set; TIO, tiotropium.

the mean CAT score was $12 \pm 6$, and $29 \%$ of patients in the TIO group and $35 \%$ in the FP/SAL group had an mMRC score $\geq 2$ (Table 2). The proportions of patients split by GOLD A-D classification were similar between the treatment groups, although the proportion of patients classified as GOLD B was slightly higher, and GOLD D slightly lower, in the FP/SAL vs the TIO group (Table 1). Regarding smoking history, 40\% were current smokers in both groups. The COPD type in most patients was characterized clinically as emphysema (Table 1). A total of 156 patients (74\% of those 210 patients in whom a value was recorded at baseline) has an eosinophil count $\geq 2 \%$.

\section{Primary outcome}

The number of patients in the FP/SAL group who continued their treatment was 136 (67\%) compared with $126(63 \%)$ patients in the TIO group; there was no statistically significant difference between the treatment groups. More than $10 \%$ of patients stepped up to triple therapy at the first assessment and thereafter there was progressive recruitment to triple therapy. The time to first switch to triple therapy was longer in the FP/SAL group compared with the TIO group, but this was not significant (log-rank test; $P=0.214$; Figure 3 ). Similarly, the odds ratio for a treatment effect was not statistically significant in both the univariate and multivariable analyses (Table 2). In both univariate and multivariable analyses, patients in GOLD groups C and D were significantly more likely to switch than those in GOLD groups A and B (Figure 4; Table 2). In univariate analyses, women, patients aged $>65$ years, and patients with higher baseline E-RS scores (Table 2) appeared to be more likely to step up. In multivariable analysis, patients in GOLD groups C and D were more likely to step up than patients in GOLD groups A and B; in addition, older age and worse E-RS chest symptoms were all significantly associated with increased likelihood to switch to triple therapy, (Table 2). Most patients (>90\%) switched owing to lack of efficacy. Three patients switched back from triple to their randomized treatment.

\section{CAT score}

In patients who did not switch, there was a general trend for improvement (Figure S1), but this was less obvious in 
Table I Patient characteristics in the mITT population

\begin{tabular}{|c|c|c|c|}
\hline Characteristics & $\begin{array}{l}\text { TIO } \\
(\mathrm{N}=20 \mathrm{I})\end{array}$ & $\begin{array}{l}\text { FP/SAL } \\
(\mathrm{N}=204)\end{array}$ & $\begin{array}{l}\text { Total } \\
(\mathrm{N}=405)\end{array}$ \\
\hline \multicolumn{4}{|l|}{ Age } \\
\hline Mean \pm SD & $68 \pm 7.12$ & $68.6 \pm 6.93$ & $68.3 \pm 7.02$ \\
\hline \multicolumn{4}{|l|}{ Age group (years), n (\%) } \\
\hline $40-49$ & $6(3)$ & $3(1)$ & $9(2)$ \\
\hline $50-59$ & $14(7)$ & $16(8)$ & $30(7)$ \\
\hline $60-69$ & $89(44)$ & $83(4 I)$ & $172(42)$ \\
\hline 70-79 & $88(44)$ & $99(49)$ & $187(46)$ \\
\hline$\geq 80$ & $4(2)$ & $3(1)$ & $7(2)$ \\
\hline \multicolumn{4}{|l|}{ Sex, n (\%) } \\
\hline Male & $193(96)$ & $192(94)$ & $385(95)$ \\
\hline \multicolumn{4}{|c|}{ History of smoking, n (\%) } \\
\hline Current smoker & $81(40)$ & $82(40)$ & $163(40)$ \\
\hline Former smoker & $119(59)$ & $122(60)$ & $24 I(60)$ \\
\hline \multicolumn{4}{|l|}{ Total pack-years } \\
\hline Mean \pm SD & $54.53 \pm 27.7$ & $60.75 \pm 33.0$ & $57.67 \pm 30.7$ \\
\hline \multicolumn{4}{|c|}{ Duration of COPD (years) } \\
\hline Mean \pm SD & $3.7 \pm 3.9$ & $3.7 \pm 4.4$ & $3.7 \pm 4.2$ \\
\hline \multicolumn{4}{|l|}{ COPD type, n (\%) } \\
\hline Chronic bronchitis & $26(13)$ & $29(14)$ & $55(14)$ \\
\hline Emphysema & $164(82)$ & $169(83)$ & $333(82)$ \\
\hline Mixed & II (5) & $6(3)$ & $17(4)$ \\
\hline \multicolumn{4}{|c|}{ Asthma overlap (current), n (\%) } \\
\hline Yes & $38(19)$ & $4 \mid(20)$ & $79(20)$ \\
\hline \multicolumn{4}{|c|}{ mMRC grade by investigator, $\mathrm{n}(\%)$} \\
\hline I & $\mid 42(7 \mid)$ & $132(65)$ & $274(68)$ \\
\hline 2 & $45(22)$ & $60(29)$ & $105(26)$ \\
\hline 3 & $14(7)$ & $12(6)$ & $26(6)$ \\
\hline 4 & 0 & 0 & 0 \\
\hline \multicolumn{4}{|l|}{$\mathrm{FEV}_{1} / \mathrm{FVC}(\%)^{\mathrm{a}}$} \\
\hline Mean \pm SD & $51.4 \pm 11.4$ & $52.6 \pm I I . I$ & $52.0 \pm 11.3$ \\
\hline \multicolumn{4}{|c|}{ CAT total score at screening } \\
\hline Mean \pm SD & $12 \pm 6$ & $12 \pm 6$ & $12 \pm 6$ \\
\hline \multicolumn{4}{|c|}{ CAT total score category, n (\%) } \\
\hline$\leq 9$ & $71(35)$ & $70(34)$ & $|4|(35)$ \\
\hline $10-15$ & $76(38)$ & $85(42)$ & $161(40)$ \\
\hline$\geq 16$ & $54(27)$ & $49(24)$ & $103(25)$ \\
\hline \multicolumn{4}{|l|}{ Predicted FEV $(\%)^{\mathrm{a}}$} \\
\hline Mean \pm SD & $57.8 \pm 13.7$ & $59.5 \pm 13.0$ & $58.7 \pm 13.3$ \\
\hline \multicolumn{4}{|c|}{ Number of exacerbations within 12 months, $\mathrm{n}(\%)$} \\
\hline 0 & $185(92)$ & $192(94)$ & $377(93)$ \\
\hline$\geq 1$ & $16(8)$ & $12(6)$ & $28(7)$ \\
\hline \multicolumn{4}{|c|}{ GOLD patient group, $\mathrm{n}(\%)$} \\
\hline A & $59(29)$ & $57(28)$ & $116(29)$ \\
\hline B & $83(4 I)$ & $100(49)$ & $183(45)$ \\
\hline $\mathrm{C}$ & $12(6)$ & $13(6)$ & $25(6)$ \\
\hline $\mathrm{D}$ & $47(23)$ & $34(17)$ & $81(20)$ \\
\hline
\end{tabular}

Note: aPost bronchodilator.

Abbreviations: CAT, COPD Assessment Test; FP/SAL, fluticasone propionate/salmeterol; GOLD, Global initiative for chronic Obstructive Lung Disease 20II criteria; mITT, modified intent-to-treat; mMRC, modified Medical Research Council; TIO, tiotropium.

those who switched. When viewing this figure, it is important to remember that higher CAT scores were one reason for switching to triple therapy and that this occurred at different times during the study.

\section{E-RS scores}

There was a large difference in E-RS total score throughout the study, between patients who remained on randomized therapy and those who switched to triple therapy (Figure 5). 
Table 2 Univariate and multivariable analyses (logistic regression) of factors responsible for switch to triple therapy

\begin{tabular}{|c|c|c|c|c|}
\hline Response & Effect & $\begin{array}{l}\text { Odds ratio } \\
\text { estimate }\end{array}$ & $95 \% \mathrm{Cl}$ & \\
\hline \multicolumn{5}{|c|}{ Univariate analysis } \\
\hline \multirow[t]{11}{*}{ Switch to TRIPLE } & Randomized treatment: FP/SAL vs TIO & 0.840 & 0.559 & 1.263 \\
\hline & GOLD patient category I: $C$ and $D$ vs $A$ and $B$ & 4.011 & 2.520 & 6.383 \\
\hline & GOLD patient category 2: $B$ and $D$ vs $A$ and $C$ & 1.790 & 1.145 & 2.796 \\
\hline & Eosinophil: $\geq 2 \%$ vs $<2 \%$ & 0.756 & 0.395 & 1.445 \\
\hline & Sex: female vs male & 2.907 & 1.160 & 7.287 \\
\hline & Age: $65-74$ vs $18-64$ years & 3.181 & $\mathrm{I} .774$ & 5.706 \\
\hline & Age: $\geq 75$ vs $18-64$ years & 4.081 & 2.030 & 8.205 \\
\hline & Smoking history: current smoker vs former smoker & 0.751 & 0.493 & 1.144 \\
\hline & RS-subscale breathlessness: mean baseline & 1.229 & 1.152 & 1.311 \\
\hline & RS-subscale cough and sputum: mean baseline & 1.213 & 1.067 & 1.379 \\
\hline & RS-subscale chest symptoms: mean baseline & 1.399 & 1.242 & 1.575 \\
\hline \multicolumn{5}{|c|}{ Multivariable analysis } \\
\hline \multirow[t]{5}{*}{ Switch to TRIPLE } & Randomized treatment (FP/SAL vs TIO) & 0.952 & 0.603 & 1.504 \\
\hline & GOLD group ( $C$ and $D$ vs $A$ and $B)$ & 3.362 & 2.047 & 5.523 \\
\hline & Age $65-74$ vs $18-64$ years & 2.862 & 1.539 & 5.323 \\
\hline & Age $\geq 75$ vs $18-64$ years & 3.141 & 1.485 & 6.643 \\
\hline & RS-subscale chest symptoms (baseline) & I.37। & 1.208 & 1.555 \\
\hline
\end{tabular}

Abbreviations: FP/SAL, fluticasone propionate/salmeterol; GOLD, Global initiative for chronic Obstructive Lung Disease 20II criteria; RS, respiratory symptoms; TIO, tiotropium.

This difference was driven by differences in breathlessness and chest symptoms, rather than cough and sputum. There was a general trend for improvement across the study period that appeared to be greater in the patients who switched to triple therapy. Analysis of E-RS total scores by baseline blood eosinophil levels showed little difference in scores between patients with high blood eosinophil counts ( $\geq 2 \%$ ) compared with low blood eosinophil counts $(<2 \%$; Figure S2). However, it should be noted that the relatively

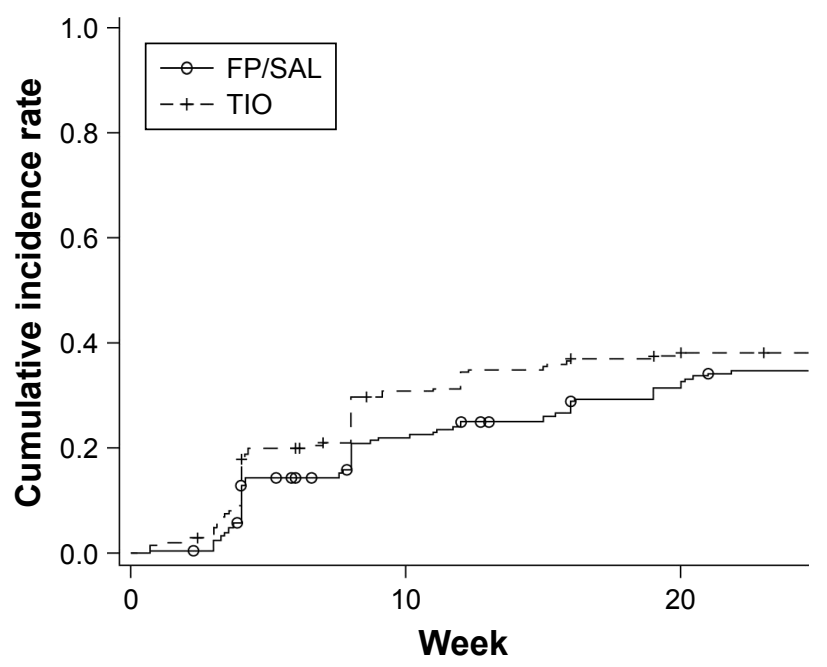

Figure 3 Kaplan-Meier curves of time to switch to triple therapy. Note: Difference in the time course between the treatment arms was not statistically significant.

Abbreviations: FP/SAL, fluticasone propionate/salmeterol; TIO, tiotropium. small numbers of patients in each subgroup, coupled with the low exacerbation rate, preclude any conclusive interpretations from these data.

\section{Time to first exacerbation}

There were very few moderate-to-severe (ie, reported) exacerbations in the patients who did not switch, but in those who stepped up to triple therapy, approximately $20 \%$ had an exacerbation by 24 weeks (Figure 6A). In contrast, $>30 \%$ of patients had at least one unreported exacerbation detected by the EXACT, even if they did not switch (Figure 6B). The Kaplan-Meier curves showed different patterns between treatment groups; the lowest incidence of EXACT events (32 patients) was seen in patients randomized to $\mathrm{FP} / \mathrm{SAL}$ who did not switch. The highest incidence of EXACT events (57 patients) was seen in those patients randomized to TIO who then switched to triple therapy (Figure 6B).

\section{Safety}

All treatments were well tolerated with AEs of any degree of severity reported by $52 \%$ (TIO-single), $71 \%$ (TIO-triple), $65 \%$ (FP/SAL-single), and $66 \%$ (FP/SAL-triple) of patients. Nasopharyngitis was the most commonly reported event in all groups (Table S2). None of the patients who were randomized to TIO and did not switch to triple therapy had an episode of pneumonia; pneumonia was reported in $2 \%$ of those randomized to, and remaining on, FP/SAL and 


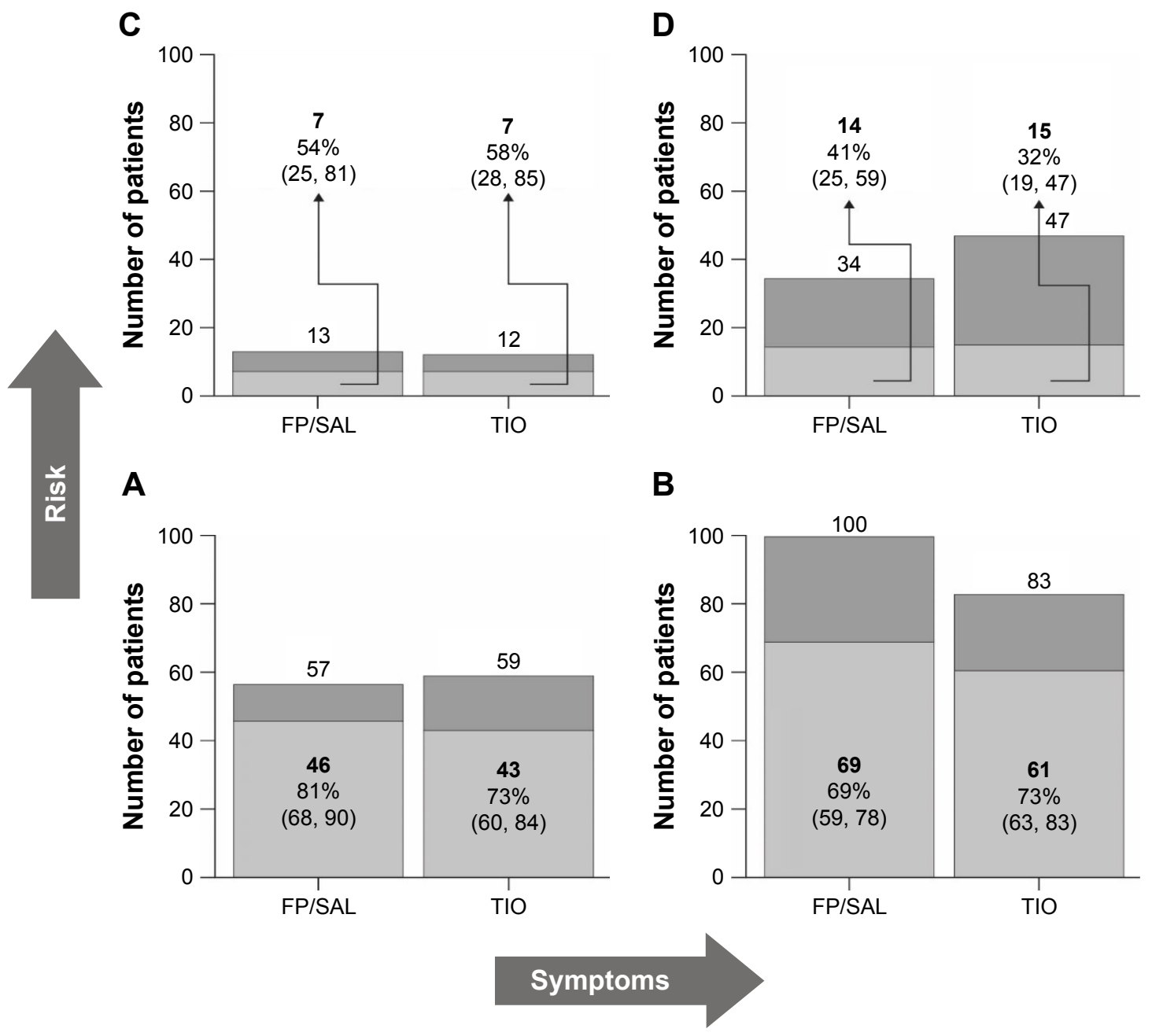

Figure 4 Analysis of the proportion of patients who continued receiving their randomized treatment in (A) GOLD group A; (B) GOLD group B; (C) GOLD group C; (D) GOLD group D.

Note: Figures in parentheses represent $95 \% \mathrm{Cls}$.

Abbreviations: FP/SAL, fluticasone propionate/salmeterol; GOLD, Global initiative for chronic Obstructive Lung Disease 20I I criteria; TIO, tiotropium.

$4 \%-8 \%$ of those who switched to triple. Two serious cases (ie, requiring hospitalization) of pneumonia occurred in each of the groups that received ICS.

\section{Discussion}

This study used an innovative trial design that allowed patients and physicians to decide whether to change treatment. In both treatment arms, patients could have their therapy stepped up, in this case by switching to triple therapy if the current treatment was considered to be insufficient. Although the physicians were given guidance about asking the patients whether they wished to step up, it remained the patients' choice. Persistence in symptoms, as measured by the CAT, or the occurrence of an exacerbation since the last visit, was the reason for switching therapy. The primary endpoint, the number of patients who remained on randomized treatment with either FP/SAL or TIO, showed that $67 \%$ of patients continued in the FP/SAL group compared with $63 \%$ of patients in the TIO group. Although the time to first switch to triple therapy was longer in patients randomized to $\mathrm{FP} / \mathrm{SAL}$, it was not significant.

There was an overall trend for patients with more severe COPD to step up their therapy, as evidenced by higher baseline symptoms and a greater risk of exacerbations. This suggests that the physicians followed the step-up protocol and the patients responded to the physicians' suggestions about stepping up therapy. The study protocol did not require baseline risk to be an indication for switching therapy, but patients at greater risk of exacerbations (GOLD 2011 groups $\mathrm{C}$ and D) were more likely to be stepped up to triple therapy during the study - either because of persisting symptoms or the occurrence of an exacerbation. The slightly 
A

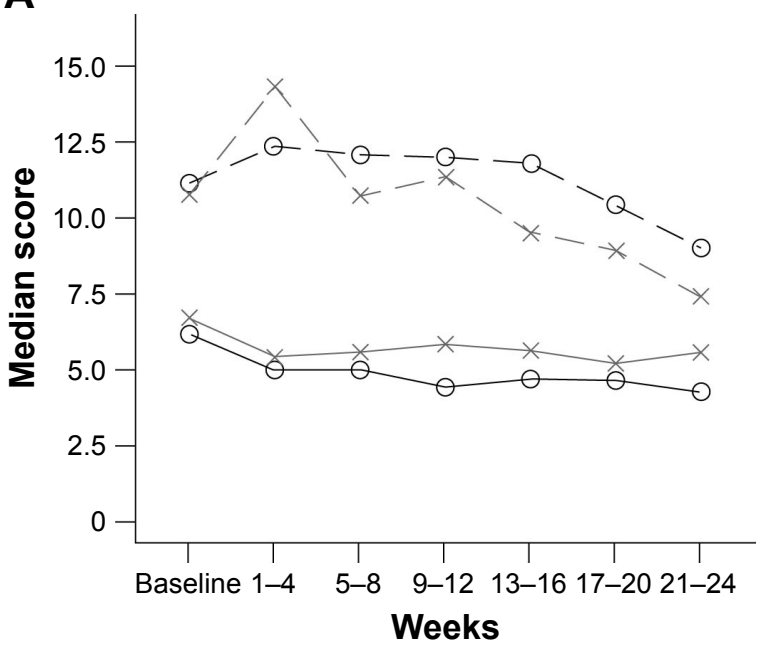

C

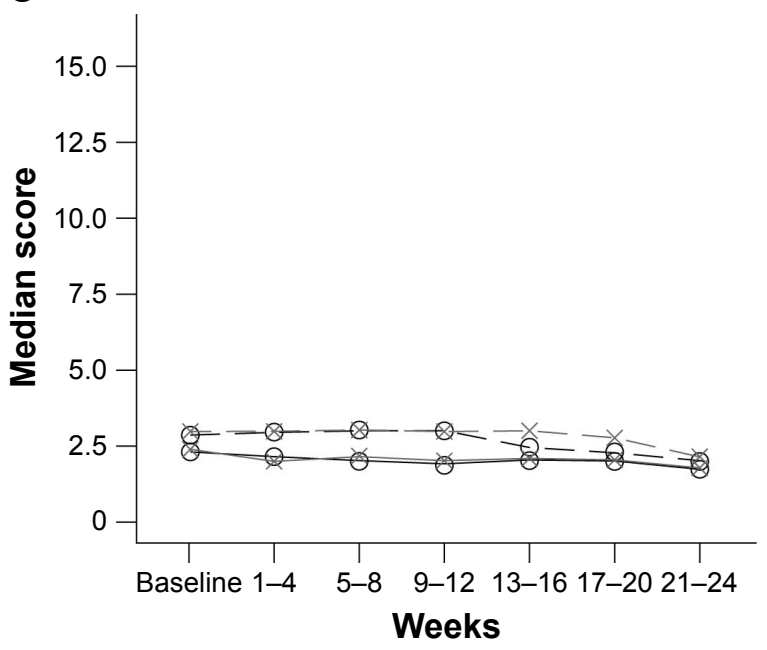

B

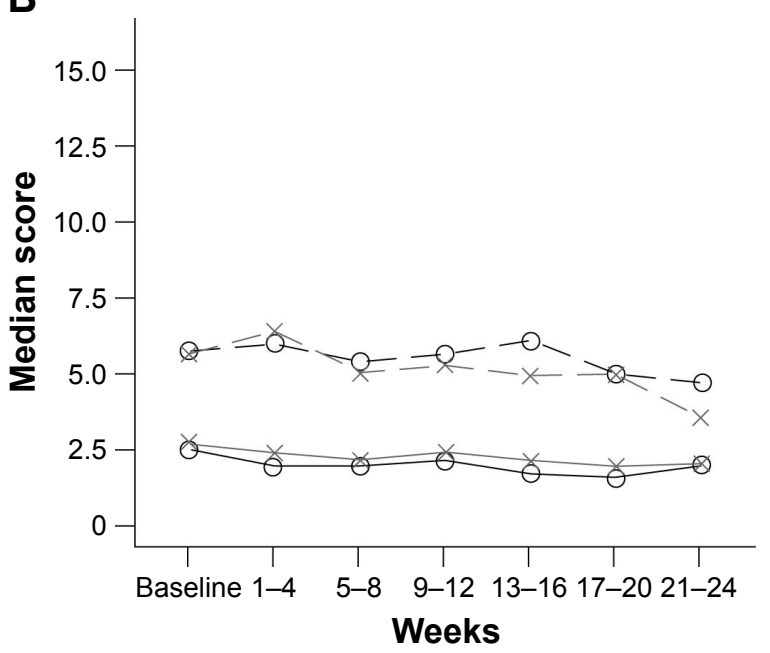

D

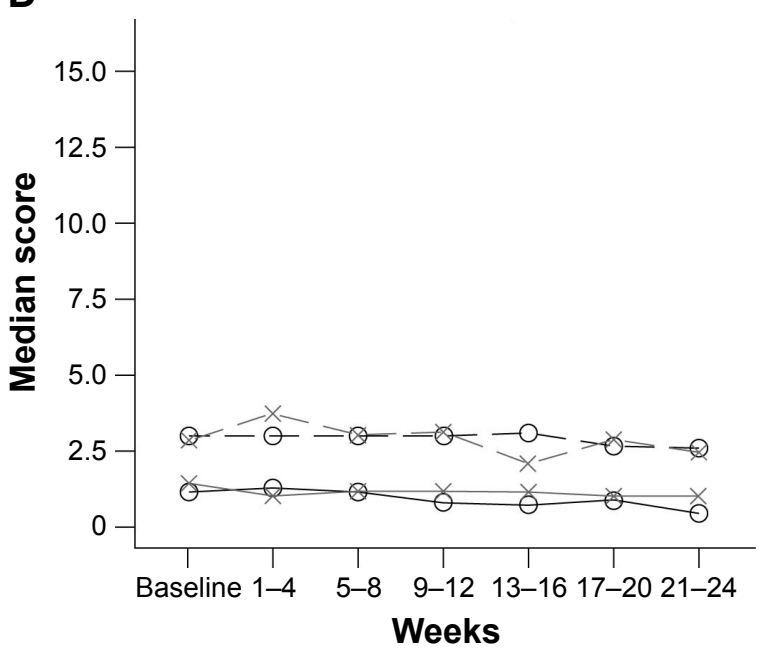

- FP/SAL single therapy $-\bigcirc$ FP/SAL triple therapy $\leftarrow$ TIO single therapy $-*-$ TIO triple therapy

Figure 5 Summary of RS median scores: (A) RS total, (B) RS breathlessness, (C) RS cough and sputum, and (D) RS chest symptoms. Abbreviations: FP/SAL, fluticasone propionate/salmeterol; RS, respiratory symptoms; TIO, tiotropium.

higher proportion of patients classified as GOLD group D in the TIO vs the FP/SAL group at baseline should be considered when comparing outcomes between treatment groups. Step-up due to an exacerbation was only suggested if the patient reported a moderate-severe exacerbation; however, the rate of reported exacerbations was very low, so opportunities for step-up were limited. Similarly, the patients generally had low CAT scores compared with those reported in primary care in Europe, ${ }^{21}$ which again may have reduced the likelihood of a switch. However, despite the relatively mild severity of the patients' COPD, the results suggest that patient management pathways in which patients and physicians make the treatment decisions can be tested in a randomized blinded controlled trial setting. This approach avoids some of the biases associated with retrospective real-world database studies, particularly confounding by severity. The study also shows that patients with more symptoms or impaired health status will take the offer of treatment step-up, which is, in itself, an important observation.

One defining characteristic of the study was that very few patients dropped out; $90 \%$ of the patients remained in the study until the end. This may be due to the use of a flexible treatment that more closely reflects clinical practice, ie, it allows for treatment intensification if the patient's condition worsens. In conventional efficacy RCTs, the patient may withdraw from the study in order to step up treatment, which therefore introduces a "healthy survivor" bias.

The study can be criticized for encouraging a more aggressive treatment approach than that seen in routine practice, in which physicians may take a more reactive 
A

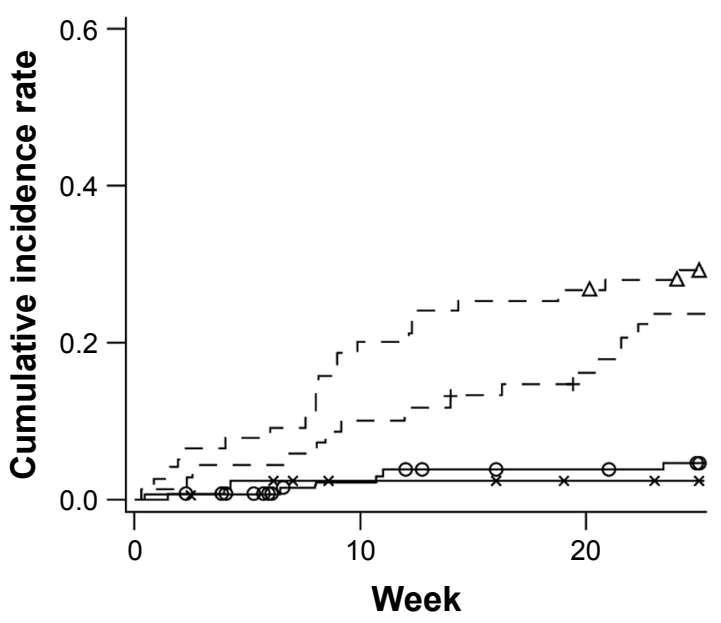

B

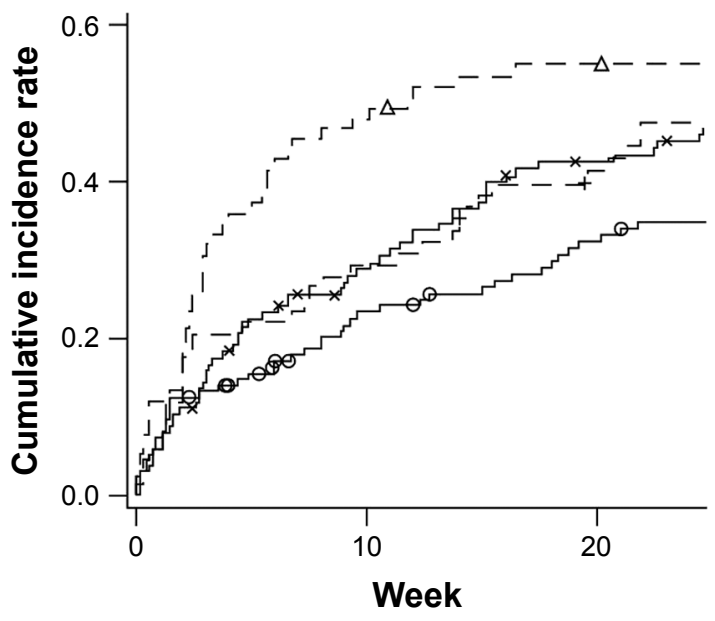

- - FP/SAL single therapy -+- FP/SAL triple therapy $\rightarrow$ - TIO single therapy $-\AA-$ TIO triple therapy

Figure 6 Kaplan-Meier curves of time to the first exacerbation defined by (A) physician's diagnosis and (B) EXACT.

Note: Differences in the time course between treatment arms were not statistically significant.

Abbreviations: EXACT, Exacerbations of COPD Tool; FP/SAL, fluticasone propionate/salmeterol; TIO, tiotropium.

approach to treatment, rather than routinely enquiring about patients' satisfaction with current treatment and offering step-up. In support of this suggestion, a UK retrospective database study showed that although patients were progressively stepped up to triple therapy (usually adding LAMA to ICS/LABA), this took a number of years..$^{22}$ Furthermore, the study shows that if physicians take a clinical evidencebased approach to increasing treatment, they appear to make appropriate decisions, since the patients who had treatment intensification were more symptomatic and at greater risk of exacerbations. This supports an observation from a retrospective study in a well-characterized cohort of Japanese patients with COPD, which showed that over half of the patients on triple therapy had their treatment intensified because of unsatisfactory improvement in symptoms when receiving their previous therapy ${ }^{23}$ The period and size of the study were too short to allow a formal test of step-up approaches in terms of outcomes such as exacerbation rate and improvements in health status, but it does show that RCTs, needed to test treatment algorithms, are feasible. In addition, the study did not assess structured approaches to stepping down treatment. The study was designed and performed before the WISDOM study on ICS withdrawal was reported ${ }^{4}$ and before GOLD suggestions that step-down could be considered; therefore, the focus was on step-up criteria. The 24-week study duration would allow little opportunity to study step-down from previous step-up, and further studies of longer duration are required to address this topic.

The choice of treatments for this study merits some discussion. This study tested an innovative methodology that attempted to model clinical practice in combination with the rigor of a clinical trial. The study was designed with the current Japanese COPD management pathway in mind; however, new trial data emerge and guidelines and treatment recommendations change, so all such trials become hostage to new developments. Although the GOLD strategy document suggests reserving ICS-containing treatments for patients at higher risk of exacerbations, ${ }^{1}$ other guidelines, such as the Japanese Respiratory Society guidelines, ${ }^{2}$ do not make that distinction. They recommend ICS/LABA as part of a treatment intensification approach in response to worsening symptoms and exercise performance as well as exacerbations. Adding an ICS to a LABA has symptomatic benefits: ${ }^{24}$ addition of ICS/LABA improved symptoms and health status when added to $\mathrm{LAMA}^{7}$ and conversely adding LAMA to ICS/LABA. ${ }^{11,25}$ The use of triple therapy is increasing, ${ }^{22}$ and although not all of it will be appropriate, some will be a suitable response by physicians to their patients' symptomatic needs. The choice of treatments for this study should also be set in historical context. At the time it was designed, dual bronchodilators were not available in Japan. Although there is evidence that LAMA/LABA and ICS/LABA have similar efficacy in terms of improvement in SGRQ score, ${ }^{26,27}$ risk-benefit considerations would now indicate use of dual bronchodilator as step-up therapy from monotherapy for symptoms, reserving ICS/LABA step-up for exacerbations.

\section{Conclusion}

Efficacy trials do not usually give guidance to physicians and guideline developers about when and how to change 
treatment. The findings from this novel trial design suggest that it is possible to perform a blinded treatment pathway RCT that minimizes bias due to the patient and physician's knowledge of initial treatment allocation. Studies of this type could provide physicians and guideline committees with a stronger evidence base on which to design and apply treatment pathways in COPD.

\section{Acknowledgments}

The authors would like to thank the investigators who participated in COSMOS-J study and the GSK COSMOS-J study team. The authors wish to acknowledge the following individuals for their contributions to the clinical study: members of the GSK team, including Akiko Terasawa, Takumi Terao, Junko Urata, Yu Jincho, Miho Hashio, Ken Tanaka, Dai Wakatabe, Toshihiro Kato, Masato Tsukada, and members of the GSK monitoring team. Editorial support in the form of development of the first draft of the manuscript, collating author comments, assembling tables and figures, and referencing was provided by Diana Jones of Cambrian Clinical Associates Ltd, and was funded by GSK. Formatting of the manuscript, tables, and figures was carried out by Matthew Robinson, DPhil, of Fishawack Communications Ltd, UK, and was funded by GSK. GSK K.K. is the funding source of this study (SCO116717; NCT01762800), and was involved in all stages of the study protocol development. GSK also took charge of all costs associated with conduct of the study, analysis of the data, and the publishing of the manuscript.

\section{Author contributions}

PWJ, MK, KF, AK, TH, HH, MHJ, GH, and TB were all involved in devising the study protocol and all authors reviewed the full data, wrote and edited the manuscript. AK was responsible for the statistical analysis of the data. All authors had full access to all of the study data, vouch for the veracity and completeness of the data and the data analysis, and had final responsibility for the decision to submit for publication. All authors contributed toward data analysis, drafting and revising the paper and agree to be accountable for all aspects of the work. The study sponsor did not place any restrictions with regard to statements made in the final paper.

\section{Disclosure}

AK is a GSK employee. MJ and $\mathrm{HH}$ are also GSK employees and have GSK stock options. TH was a GSK employee during the conduct of this study and development of this manuscript and is now employed by Pfizer Japan Inc., Japan. GH previously worked for GSK and received a consultancy fee from
GSK during the conduct of this study and development of this manuscript. PWJ discloses being now employed by GSK; prior to this and during the conduct of the study, he received personal fees from GSK for engagement with this study and from AstraZeneca and Novartis, outside of the submitted work, but in related fields. The authors report no other conflicts of interest in this work.

\section{References}

1. Global Initiative for Chronic Obstructive Lung Disease (GOLD). Global Strategy for the Diagnosis, Management and Prevention of COPD. 2017. Available from: http://goldcopd.org/gold-2017-global-strategydiagnosis-management-prevention-copd/. Accessed March 29, 2017.

2. Japanese Respiratory Society. Guidelines for the Diagnosis and Treatment of COPD. 4th ed; 2013. Available from: https://www.jrs.or.jp/ modules/guidelines/index.php?content_id=1. Accessed March 29, 2017.

3. National Institute for Health and Clinical Excellence. NICE clinical guideline 101. Available from: http://www.nice.org.uk/nicemedia/ live/13029/49397/49397. Accessed May 5, 2016.

4. Magnussen H, Watz H, Kirsten A, et al. Stepwise withdrawal of inhaled corticosteroids in COPD patients receiving dual bronchodilation: WISDOM study design and rationale. Respir Med. 2014;108(4): 593-599.

5. Nannini LJ, Poole P, Milan SJ, Holmes R, Normansell R. Combined corticosteroid and long-acting beta ${ }_{2}$-agonist in one inhaler versus placebo for chronic obstructive pulmonary disease. Cochrane Database Syst Rev. 2013;11:CD003794.

6. Karner C, Chong J, Poole P. Tiotropium versus placebo for chronic obstructive pulmonary disease. Cochrane Database Syst Rev. 2012;7: CD009285.

7. Welte T, Miravitlles M, Hernandez P, et al. Efficacy and tolerability of budesonide/formoterol added to tiotropium in patients with chronic obstructive pulmonary disease. Am J Respir Crit Care Med. 2009; 180(8):741-750.

8. Siler TM, Kerwin E, Sousa AR, Donald A, Ali R, Church A. Efficacy and safety of umeclidinium added to fluticasone furoate/vilanterol in chronic obstructive pulmonary disease: results of two randomized studies. Respir Med. 2015;109(9):1155-1163.

9. Saito T, Takeda A, Hashimoto K, Kobayashi A, Hayamizu T, Hagan GW. Triple therapy with salmeterol/fluticasone propionate $50 / 250$ plus tiotropium bromide improve lung function versus individual treatments in moderate-to-severe Japanese COPD patients: a randomized controlled trial - evaluation of Airway sGaw after treatment with tripLE. Int J Chron Obstruct Pulmon Dis. 2015;10:2393-2404.

10. Perng DW, Wu CC, Su KC, Lee YC, Perng RP, Tao CW. Additive benefits of tiotropium in COPD patients treated with long-acting beta agonists and corticosteroids. Respirology. 2006;11(5):598-602.

11. Frith PA, Thompson PJ, Ratnavadivel R, et al. Glycopyrronium oncedaily significantly improves lung function and health status when combined with salmeterol/fluticasone in patients with COPD: the GLISTEN study, a randomised controlled trial. Thorax. 2015;70(6):519-527.

12. Cazzola M, Matera MG. Triple combinations in chronic obstructive pulmonary disease - is three better than two? Expert Opin Pharmacother. 2014;15(17):2475-2478.

13. Aaron SD, Vandemheen KL, Fergusson D, et al. Tiotropium in combination with placebo, salmeterol, or fluticasone-salmeterol for treatment of chronic obstructive pulmonary disease: a randomized trial. Ann Intern Med. 2007;146(8):545-555.

14. Short PM, Williamson PA, Elder DHJ, Lipworth SIW, Schembri S, Lipworth BJ. The impact of tiotropium on mortality and exacerbations when added to inhaled corticosteroids and long-acting $\beta$-agonist therapy in COPD. Chest. 2012;141(1):81-86. 
15. Jones PW, Kato M, Fujimoto K, et al. COPD Symptom Based Management and Optimize Treatment Strategy in Japan (The COSMOS-J Trial). Am J Respir Crit Care Med. 2016;193:A7906.

16. Vestbo J, Hurd SS, Agustí AG, et al. Global strategy for the diagnosis, management, and prevention of chronic obstructive pulmonary disease: GOLD executive summary. Am J Respir Crit Care Med. 2013;187(4): 347-365.

17. Betsuyaku T, Kato M, Fujimoto K, et al. A study to assess COPD Symptom-based Management and to Optimise treatment Strategy in Japan (COSMOS-J) based on GOLD 2011. Int J Chron Obstruct Pulmon Dis. 2013;8:453-459.

18. Jones PW, Harding G, Berry P, Wiklund I, Chen WH, Kline Leidy N. Development and first validation of the COPD Assessment Test. Eur Respir J. 2009;34(3):648-654.

19. Leidy NK, Wilcox TK, Jones PW, et al. Standardizing measurement of chronic obstructive pulmonary disease exacerbations. Reliability and validity of a patient-reported diary. Am J Respir Crit Care Med. 2011; 183(3):323-329.

20. Leidy NK, Murray LT, Monz BU, et al. Measuring respiratory symptoms of COPD: performance of the EXACT- Respiratory Symptoms Tool (E-RS) in three clinical trials. Respir Res. 2014;15:124.

21. Jones PW, Brusselle G, dal Negro RW, et al. Properties of the COPD assessment test in a cross-sectional European study. Eur Respir J. 2011 38(1):29-35
22. Brusselle G, Price D, Gruffydd-Jones K, et al. The inevitable drift to triple therapy in COPD: an analysis of prescribing pathways in the UK. Int J Chron Obstruct Pulmon Dis. 2015;10:2207-2217.

23. Miyazaki M, Nakamura H, Takahashi S, et al. The reasons for triple therapy in stable COPD patients in Japanese clinical practice. Int $J$ Chron Obstruct Pulmon Dis. 2015;10:1053-1059.

24. Kew KM, Dias S, Cates CJ. Long-acting inhaled therapy (beta-agonists, anticholinergics and steroids) for COPD: a network meta-analysis. Cochrane Database Syst Rev. 2014;3:CD010844.

25. Siler TM, Kerwin E, Tombs L, Fahy WA, Naya I. Triple therapy of umeclidinium + inhaled corticosteroids/long-acting beta2 agonists for patients with COPD: pooled results of randomized placebo-controlled trials. Pulm Ther. 2016;2(1):43-58.

26. Vogelmeier CF, Bateman ED, Pallante J, et al. Efficacy and safety of once-daily QVA149 compared with twice-daily salmeterol-fluticasone in patients with chronic obstructive pulmonary disease (ILLUMINATE): a randomised, double-blind, parallel group study. Lancet Respir Med. 2013;1(1):51-60.

27. Singh D, Worsley S, Zhu CQ, Hardaker L, Church A. Umeclidinium/ vilanterol versus fluticasone propionate/salmeterol in COPD: a randomised trial. BMC Pulm Med. 2015;15:91. 


\section{Supplementary materials}

Table SI Institutional review boards at participating study sites

\begin{tabular}{|c|c|c|}
\hline \multicolumn{2}{|c|}{ Site information } & \multirow[t]{2}{*}{ Name of institutional review board } \\
\hline & Prefecture & \\
\hline I & Hokkaido & National Hospital Organization Central Review Board \\
\hline 2 & Ibaraki & National Hospital Organization Central Review Board \\
\hline 3 & Ibaraki & Tsuchiura Kyodo General Hospital IRB \\
\hline 4 & Ibaraki & Ibaraki Prefectural Central Hospital IRB \\
\hline 5 & Ibaraki & Review Board of Human Rights and Ethics for Clinical Studies IRB \\
\hline 6 & Tokyo & Tokyo-Eki Center-building Clinic IRB \\
\hline 7 & Tokyo & Suzuki Clinic Internal Medicine and Cardiology IRB \\
\hline 8 & Tokyo & Showa General Hospital IRB \\
\hline 9 & Kanagawa & Yasuda Hospital IRB \\
\hline 10 & Kanagawa & Sugiura Clinic IRB \\
\hline II & Niigata & Niigata Rinko Hospital IRB \\
\hline 12 & Niigata & Niigata City General Hospital IRB \\
\hline 13 & Niigata & National Hospital Organization Central Review Board \\
\hline 14 & Shizuoka & Review Board of Human Rights and Ethics for Clinical Studies IRB \\
\hline 15 & Kyoto & Japanese Red Cross Kyoto Daini Hospital IRB \\
\hline 16 & Kyoto & National Hospital Organization Central Review Board \\
\hline 17 & Osaka & Chuto Hospital IRB \\
\hline 18 & Osaka & National Hospital Organization Central Review Board \\
\hline 19 & Nara & Nara Hospital Kinki University Faculty of Medicine IRB \\
\hline 20 & Hyogo & Terada Clinic Internal Respiratory Medicine IRB \\
\hline 21 & Hiroshima & National Hospital Organization Central Review Board \\
\hline 22 & Hiroshima & Kure Kyosai Hospital-Hiroshima IRB \\
\hline 23 & Hiroshima & Chugoku Central Hospital-Hiroshima IRB \\
\hline 24 & Yamaguchi & National Hospital Organization Central Review Board \\
\hline 25 & Kagawa & Yasuda Hospital IRB \\
\hline 26 & Kagawa & KKR Takamatsu Hospital IRB \\
\hline 27 & Kagawa & Takamatsu Municipal Hospital IRB \\
\hline 28 & Kochi & Sugiura Clinic IRB \\
\hline 29 & Fukuoka & Fukuoka University Hospital IRB \\
\hline 30 & Fukuoka & Sugiura Clinic IRB \\
\hline 31 & Saga & Saga-Ken Medical Center Koseikan IRB \\
\hline 32 & Okinawa & Chuto Hospital IRB \\
\hline 33 & Okinawa & Chuto Hospital IRB \\
\hline 34 & Okinawa & Okinawa Prefectural Chubu Hospital IRB \\
\hline 35 & Ibaraki & Ibaraki Higashi National Hospital IRB \\
\hline 36 & Osaka & AMC Nishi-umeda Clinic IRB \\
\hline 37 & Tokyo & AMC Nishi-umeda Clinic IRB \\
\hline 38 & Osaka & Clinical Research Tokyo Hospital IRB \\
\hline 39 & Hiroshima & Hiroshima Prefectural Hospital IRB \\
\hline
\end{tabular}

Abbreviation: IRB, institutional review board. 
A

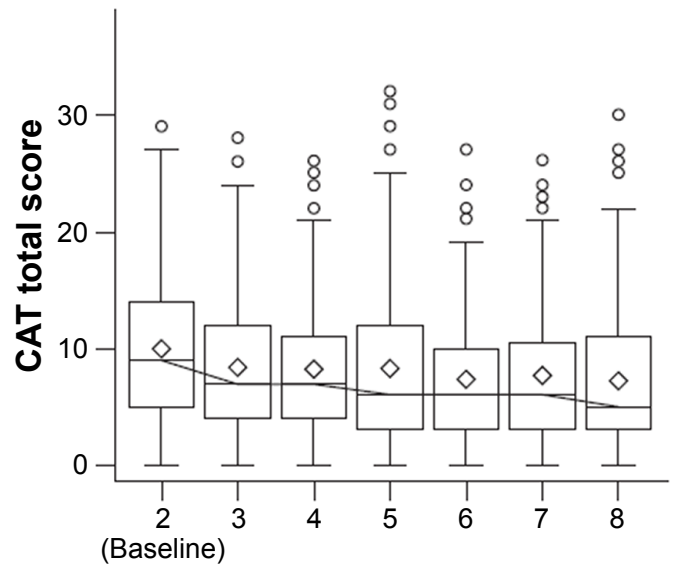

Visit

C

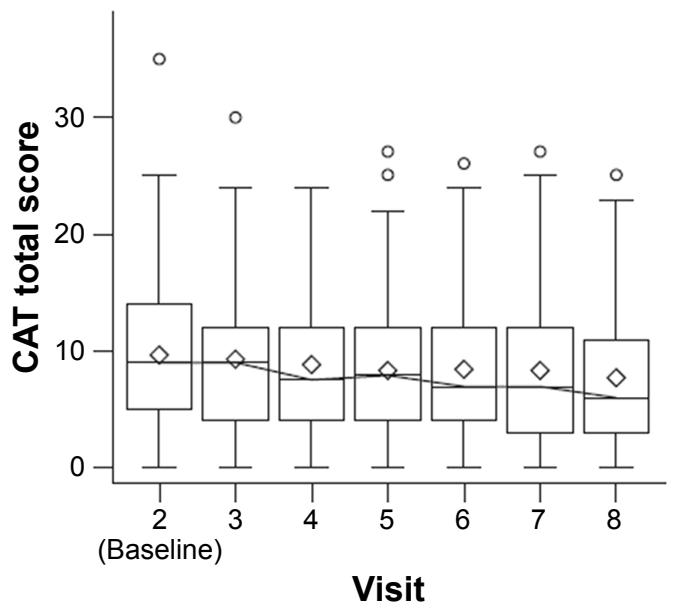

B

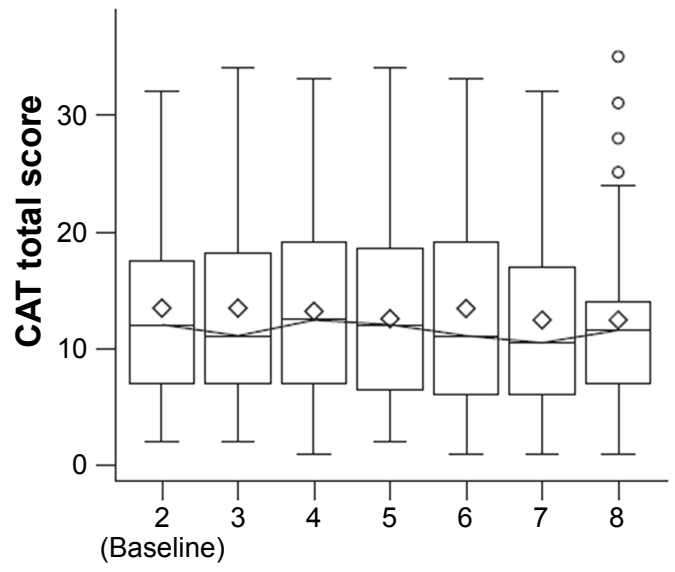

Visit

D

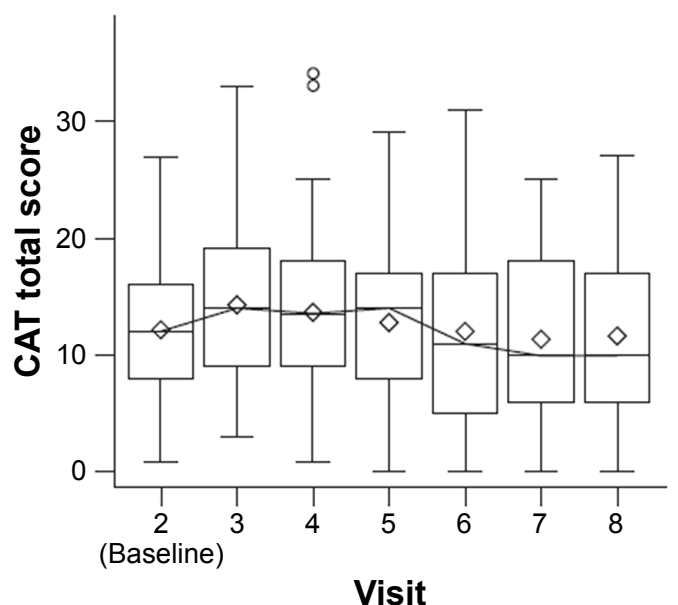

Figure SI Summary of CAT total score for patients who received (A) FP/SAL single therapy; (B) FP/SAL triple therapy; (C) TIO single therapy; and (D) TIO triple therapy.

Abbreviations: CAT, COPD assessment test; FP/SAL, fluticasone propionate/salmeterol; TIO, tiotropium.

A

FP/SAL single $(\mathrm{N}=\mathbf{2 1})$

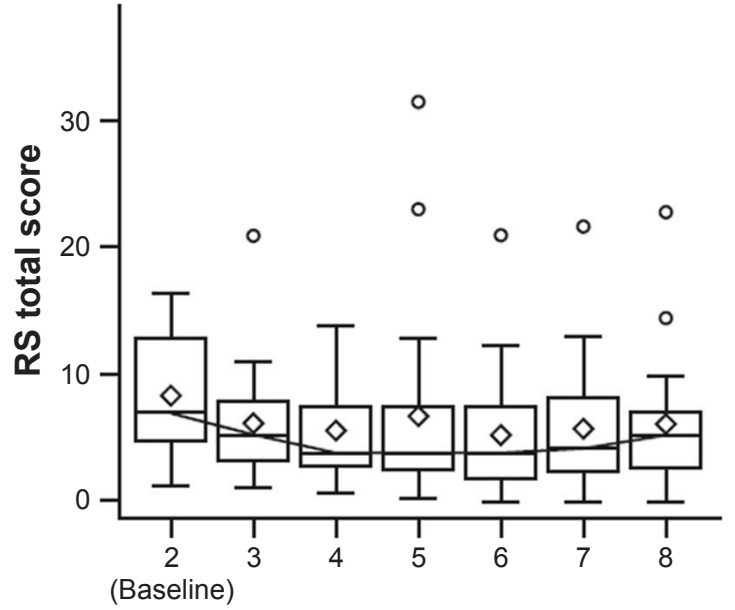

Visit
FP/SAL triple $(\mathrm{N}=10)$

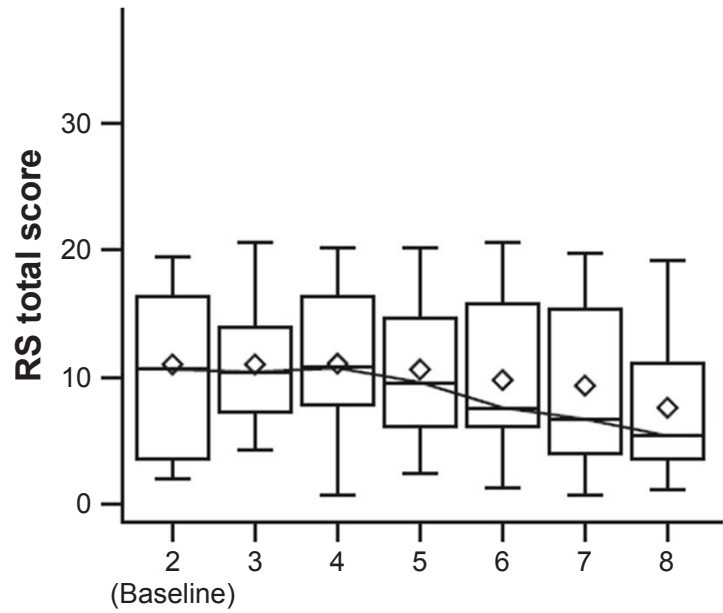

Visit

Figure S2 (Continued) 
TIO single $(\mathrm{N}=13)$

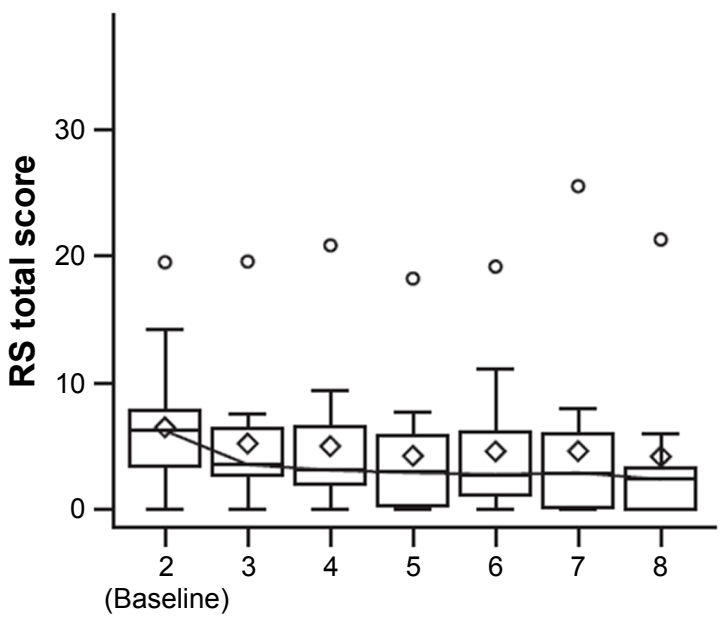

Visit

B FP/SAL single $(\mathrm{N}=56)$

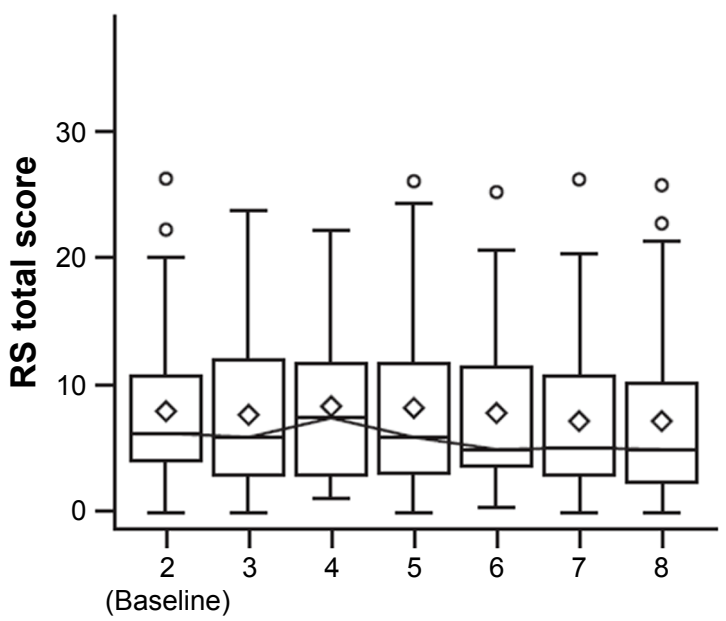

Visit

TIO single $(\mathrm{N}=52)$

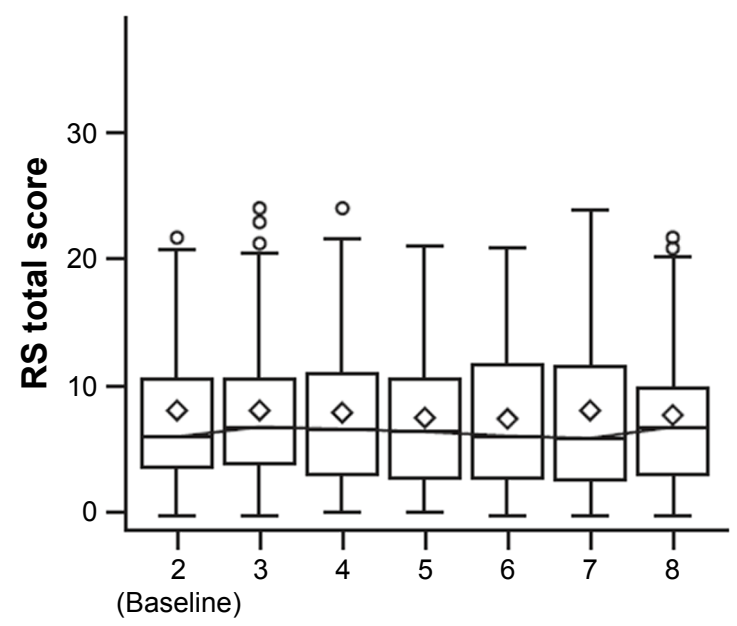

Visit
TIO triple $(\mathrm{N}=10)$

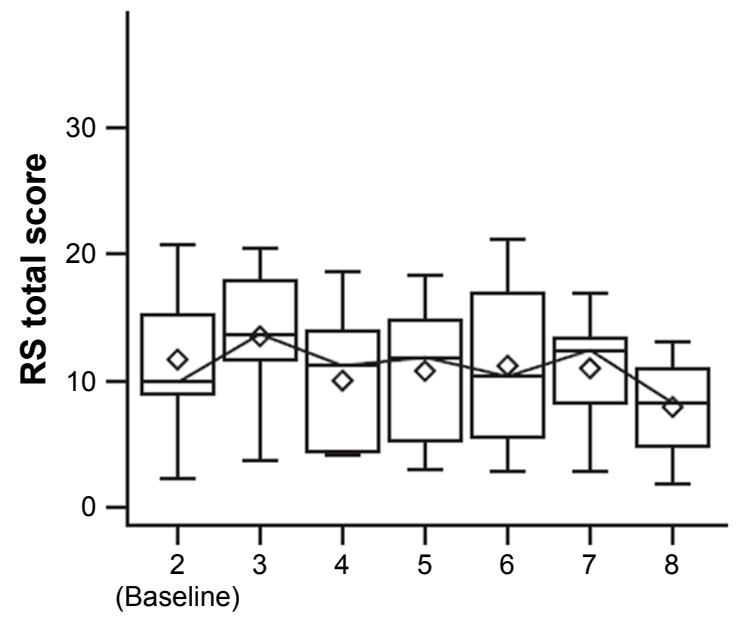

Visit

FP/SAL triple $(\mathrm{N}=25)$

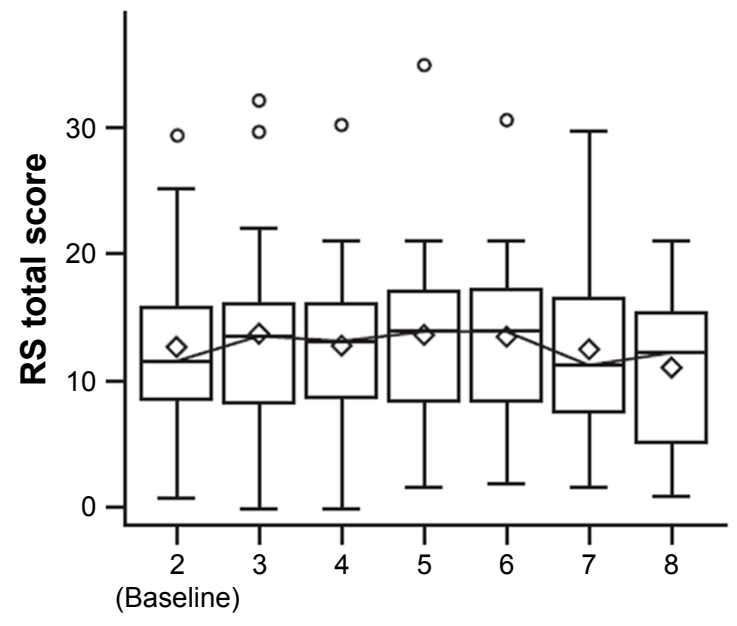

Visit

TIO triple $(\mathrm{N}=\mathbf{2 3})$

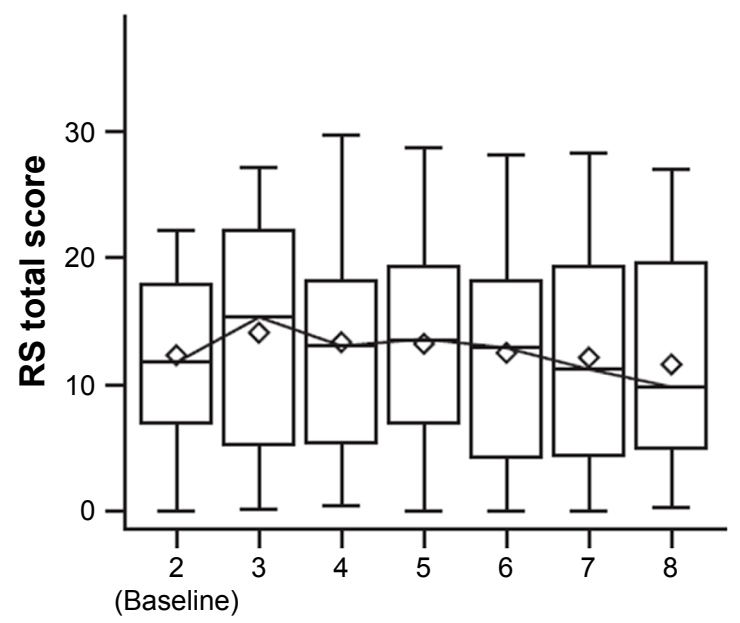

Visit

Figure S2 Summary of E-RS total scores by treatment group for patients with (A) low blood eosinophil counts ( $<2 \%)$ and $(\mathbf{B})$ high blood eosinophil counts $(\geq 2 \%)$ at baseline. Abbreviations: E-RS, EXACT-Respiratory Symptoms; FP/SAL, fluticasone propionate/salmeterol; RS, Respiratory Symptoms; TIO, tiotropium. 
Table S2 Summary of adverse and serious adverse events ( $\geq 5$ patients)

\begin{tabular}{|c|c|c|c|c|}
\hline & $\begin{array}{l}\text { TIO-single } \\
(\mathbf{N}=126)\end{array}$ & $\begin{array}{l}\text { TIO-triple } \\
(\mathrm{N}=75)\end{array}$ & $\begin{array}{l}\text { FP/SAL-single } \\
(\mathrm{N}=136)\end{array}$ & $\begin{array}{l}\text { FP/SAL-triple } \\
(\mathrm{N}=68)\end{array}$ \\
\hline Any AE, n (\%) & $65(52)$ & $53(7 I)$ & $88(65)$ & $45(66)$ \\
\hline Nasopharyngitis & $19(15)$ & $20(27)$ & $25(18)$ & $14(2 \mid)$ \\
\hline Bronchitis & $7(6)$ & $5(7)$ & $7(5)$ & $7(10)$ \\
\hline Oral candidiasis & 0 & $2(3)$ & $3(2)$ & $3(4)$ \\
\hline Pneumonia & 0 & $6(8)$ & $3(2)$ & $3(4)$ \\
\hline Drug-related events, n (\%) & $10(8)$ & $8(\mathrm{II})$ & $29(2 I)$ & $9(13)$ \\
\hline AEs leading to withdrawal, $n$ (\%) & $6(5)$ & $3(4)$ & $13(10)$ & 0 \\
\hline Any SAE, n (\%) & $8(6)$ & $8(\mathrm{II})$ & $8(6)$ & $6(9)$ \\
\hline Pneumonia & 0 & $2(3)$ & $2(1)$ & $2(3)$ \\
\hline COPD & $\mathrm{I}(<\mathrm{I})$ & $3(4)$ & $I(<I)$ & 0 \\
\hline
\end{tabular}

Abbreviations: $\mathrm{AE}$, adverse event; FP/SAL, fluticasone propionate/salmeterol; SAE, serious adverse event; TIO, tiotropium.

\section{Publish your work in this journal}

The International Journal of COPD is an international, peer-reviewed journal of therapeutics and pharmacology focusing on concise rapid reporting of clinical studies and reviews in COPD. Special focus is given to the pathophysiological processes underlying the disease, intervention programs, patient focused education, and self management protocols.

\section{Dovepress}

This journal is indexed on PubMed Central, MedLine and CAS. The manuscript management system is completely online and includes a very quick and fair peer-review system, which is all easy to use. Visit http://www.dovepress.com/testimonials.php to read real quotes from published authors.

Submit your manuscript here: http://www.dovepress.com/international-journal-of-chronic-obstructive-pulmonary-disease-journal 